\title{
Propuesta de un modelo de interfaz genérica para sistemas de recuperación de información
}

\author{
Vivina Asensi-Artiga \\ Departamento de Información y Documentación \\ Universidad de Murcia \\ Juan-Antonio Pastor-Sánchez \\ Servicio de Información \\ Universidad de Murcia
}

\subsection{Resumen}

Se presenta un modelo teórico de una interfaz de usuario de propósito general para la recuperación de información. Se revisan las propuestas realizadas por diferentes autores sobre las características funcionales que deben reunir las interfaces de sistemas de recuperación de información, teniendo en cuenta que pueden operar con información de naturaleza muy diversa (sistemas de hipertexto, OPAC, bases de datos en CD-ROM, etc.). Se proponen unas directrices que deben guiar el diseño de dichas interfaces de usuario.

Palabras Clave: Intefaces de usuario, Sistemas de Recuperación de Información. Sistemas hipermedia.

\subsection{Abstract}

A general-purpose interface model for information retrieval systems is proposed. A revision of the literature in the subject is made, studying the proposals of different authors about the functional characteristics that such interfaces must accomplish, taking into account that they operate with information of different nature (hypertext systems, OPAC, CD-ROM Databases, etc.). Some guidelines for their design are suggested.

Keywords: Interfaces. Information Retrieval Systems. Hypermedia systems. 


\section{Introducción}

En un sistema de recuperación de información (SRI), la interfaz constituye un elemento fundamental, por la importancia que adquiere para que el usuario sea capaz de obtener los beneficios que le ofrece el sistema. Para el estudio de las características de una interfaz hay que tener en cuenta tanto su estructura, para la organización de la información, como las posibilidades de representación de esta información para el usuario.

En el campo de la Recuperación de la Información, los trabajos realizados en relación con la evaluación de interfaces sobre diferentes aplicaciones, muestran los avances conseguidos y también el camino que queda por recorrer. Estos trabajos han permitido llevar a cabo estudios comparativos de los diferentes modelos evaluados, así como la propuesta de otros modelos que supongan un avance en el campo de la interacción hombre-máquina, para la recuperación de información, de manera satisfactoria para el usuario.

Los conceptos de relevancia, utilidad, satisfacción, pertinencia, exhaustividad ... son utilizados habitualmente en los trabajos realizados sobre recuperación de información. Pero estos conceptos requieren especial atención por cuanto deben utilizarse en un entorno real, de manera que el usuario sea capaz de comprender su significado, y de aplicarlo si es necesario para la evaluación del sistema de recuperación que utilice habitualmente.

Actualmente, la tecnología permite ampliar las funciones del interfaz hasta límites insospechados. Las posibilidades del hipertexto para expandir los canales de comunicación posibles, en ocasiones nos desborda. El desarrollo de interfaces inteligentes, las aplicaciones de los sistemas multimedia y la introducción de elementos cada vez más semejantes al intermediario humano - los intermediarios inteligentes, desarrollados en el campo de los sistemas expertos- amplían el horizonte para el diseño de nuevos modelos.

\section{Metodología}

La investigación para el planteamiento de un modelo genérico de inferfaz de usuario para la recuperación de información se ha desarrollado en las siguientes fases:

a) Delimitación conceptual de la terminología utilizada habitualmente en el campo de la Recuperación de Información.

b) Análisis de las características generales que debe reunir un modelo de interfaz, partiendo de los estudios publicados en los últimos diez años.

c) Estudio de las posibilidades del hipertexto para la recuperación de información. 
d) Propuesta de un modelo de interfaz.

\subsection{Delimitación de Conceptos}

El proceso de búsqueda de información implica la aplicación de una estrategia determinada (Pejtersen, 1991, p. 8) en relación con la forma en que el usuario y el sistema categorizan la información durante el proceso de recuperación, mediante la identificación y formulación de necesidades de información del usuario.

Marchinioni (1992) ofrece una aproximación general a los interfaces mediante la descripción de las operaciones realizadas en los procesos de búsqueda y recuperación de información, representados en el siguiente diagrama (Fig. 1).

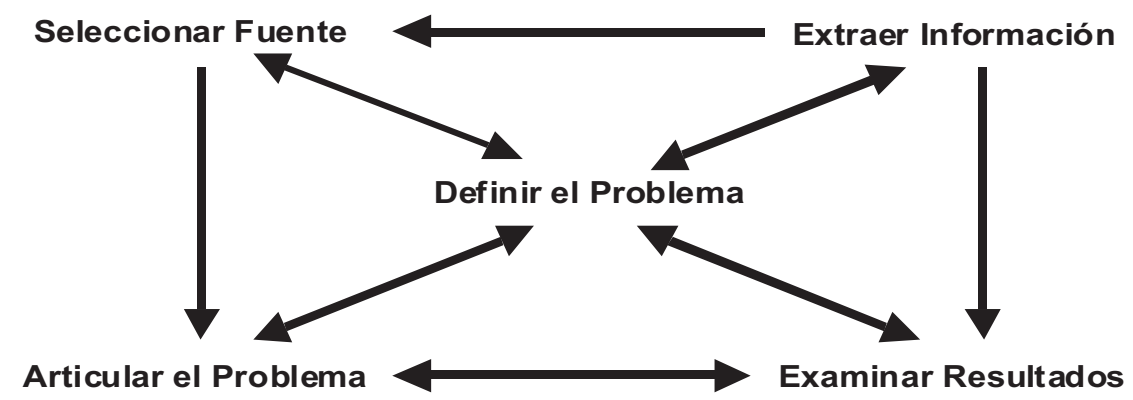

Fig. 1. Operaciones en la Búsqueda de Información

Puesto que el usuario se encarga de iniciar una búsqueda y retroalimentarla a partir de los resultados obtenidos, es evidente que la interfaz desempeña un papel fundamental, ya que se trata del elemento catalizador entre usuario y sistema. Además, hay que tener en cuenta que el principal objetivo del SRI durante la búsqueda de información consiste en ofrecer resultados exhaustivos y precisos.

Aunque en este trabajo utilizaremos el término 'exhaustividad' (recall, en inglés), consideramos oportuno mencionar otras traducciones aceptadas en español para expresar el mismo concepto, a saber 'llamada' (rappel, en francés) o 'cobertura'. Cuando se realiza una búsqueda, los documentos recuperados se quedan divididos en dos subconjuntos: recuperados y no recuperados. Además, todos los documentos (recuperados o no) se subdividen a su vez en documentos 
pertinentes y no pertinentes (según el criterio del usuario). Esto último se puede representar por medio del siguiente esquema ofrecido por Lancaster (1978) (Fig. 2).

La precisión puede considerarse como un tipo de factor coste: el tiempo que el usuario necesita para separar las referencias pertinentes de las que no lo son (Láncaster, 1978). El índice de precisión indica la capacidad del sistema para rechazar documentos no relevantes, y el índice de exhaustividad, la capacidad para proporcionar todos los documentos relevantes para una solicitud, conteni-

\begin{tabular}{|c|c|c|c|}
\hline Documento & Relevante & No relevante & Total \\
\hline Recuperado & $\mathrm{a}$ & $\mathrm{b}$ & $\mathrm{a}+\mathrm{b}$ \\
\hline No recuperado & $\mathrm{c}$ & $\mathrm{d}$ & $\mathrm{c}+\mathrm{d}$ \\
\hline Total & $\mathrm{a}+\mathrm{c}$ & $\mathrm{b}+\mathrm{d}$ & $\mathrm{a}+\mathrm{b}+\mathrm{c}+\mathrm{d}$ \\
\hline
\end{tabular}

Fig. 2. Evaluación de las búsquedas (Lancaster, 1978)

dos en la memoria del sistema (Abad, 1997). Para Blair (1990), la precisión indica la proporción de documentos recuperados que se juzgan útiles para el usuario.

Consideramos que los resultados de una búsqueda son satisfactorios cuando se recuperan todos los documentos contenidos en la base de datos que el usuario juzga como relevantes $(\mathrm{a}+\mathrm{c})$, rechazando por tanto, los que juzga como no relevantes $(b+d)$. En este caso obtendríamos una respuesta con el grado más alto posible tanto de exhaustividad como de precisión en la recuperación, basándonos en las siguientes fórmulas:

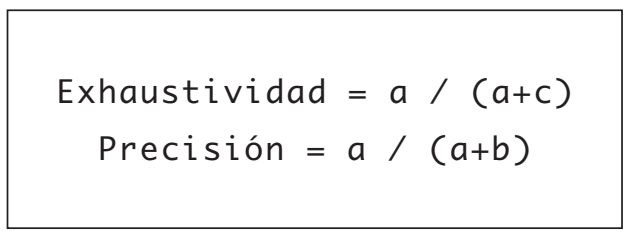

Scire. $4: 1$ (en.-jun. 1998) 71-88 
Generalmente, puede deducirse que cuando se produce una exhaustividad baja en la recuperación, el silencio documental es alto, mientras que el ruido es muy bajo. En cambio, una exhaustividad alta en la recuperación proporciona poco silencio, pero bastante ruido documental.

Objetivamente, el concepto de relevancia es muy difícil de definir (Blair, 1990), incluso para los mismos investigadores. Un documento puede ser relevante para una determinada búsqueda y en un momento preciso, sin que ello implique necesariamente la descripción de un procedimiento general para emitir un juicio de relevancia a partir de los documentos recuperados para un perfil de búsqueda.

Abad (1997), Manglano y Handcock-Beaulieu (1995), y Blair (1990) asocian la relevancia de un documento a su utilidad. Blair, citando a Cooper, advierte que el usuario en realidad lo que desea obtener es información útil. Utilidad es un concepto más amplio, que incluye, además del concepto de "temas relacionados", el de cualidad, novedad, importancia, credibilidad y otras características equivalentes a la recuperación de documentos que satisfagan su necesidad porque los considere apropiados en ese momento.

\subsection{Sistemas Hipermedia y Recuperación de Información}

La recuperación de la información depende en gran medida del tratamiento que ha recibido previamente a la búsqueda por parte del usuario. Cuando hablamos de sistemas de recuperación de información, también incluimos a los sistemas de hipertexto, desde el punto de vista de los procesos de navegación/consulta.

Las características propias de la interfaz de un sistema de información implican que el estudio de esta aplicación se realice en su conjunto, considerándola como parte del sistema de información y no únicamente como la parte de representación gráfica on-line de esta información. Dentro del concepto de interfaz, siguiendo el esquema de Kerr (1987), cabe distinguir: estructura inmediata; estructura interna; estructura externa, y contexto físico y psicológico del usuario (conjunto de conocimientos necesarios para desenvolverse en el medio).

Algunos autores (Martínez, 1998) apuntan que los documentos hipermedia se crean a través de un doble proceso de estructuración. Por una parte, se crean la estructuras espaciales y temporales que le conferirán al documento su aspecto de presentación. Por otra, se establecen una serie de enlaces de hipertexto que estructuran el contenido de los diversos elementos informativos.

El hipertexto, en sus múltiples aplicaciones, constituye un entorno ideal para la recuperación de información (Marchinioni, 1992), ya que los procesos de búsqueda se realizan de un modo más comprensivo para el usuario, mediante la acti- 
vación y seguimiento de enlaces de forma interactiva. Lee (1997) presenta un modelo de datos, un lenguaje de consulta y un esquema de indización para documentos hipermedia, lo que nos ofrece un enriquecimiento de ambos campos: los documentos hipermedia y los SRI.

Tampoco hay que olvidar que el hipertexto constituye una "metainterfaz" para la consulta de bases de datos (Croft y Turtle, 1989), mediante el establecimiento de enlaces dinámicos de hipertexto que constituyen una serie de consultas que se ejecutan a través de la activación de los mismos.

Debido a las posibilidades del hipertexto para la recuperación de información, el diseño de un modelo de interfaz navegacional permitirá resolver problemas latentes en el sistema. Generalmente, estos problemas están relacionados con la orientación, lenguaje de recuperación (sintaxis), rapidez de acceso a los documentos ya recuperados anteriormente y almacenamiento de sus localizaciones respectivas, y visualización del destino de los diferentes enlaces por los que puede optar en cada momento.

Además, del mismo modo que un sistema de recuperación de información convencional incluye un histórico de búsquedas, el interfaz de hipertexto incluirá un histórico de navegación tanto a través de la red semántica como de la red de documentos (Rada, 1991). Por último, como desarrollo del sistema de hipertexto, el interfaz debe permitir la posibilidad de establecer enlaces para activar diferentes motores de búsqueda y de otros sistemas de recuperación.

Pastor y Saorín (1995) presentan un modelo doble de interfaz navegacional basado en el hipertexto. En este modelo, abordan la integración de diversas herramientas de navegación (browsing, redes semánticas, registros de marcas, funciones de recuperación de información, etc., y la estructuración de la información a partir de una tipología de enlaces.

Catenazzi y Sommaruga (1994) aportan un modelo de interfaz para libros electrónicos. En este modelo, en el nivel de servicios para el lector presentan una serie de herramientas que combinan la lectura con la búsqueda y recuperación de información: herramientas de navegación, orientación, históricos de consulta, personalización de la interfaz, búsqueda de información y acceso a recursos externos.

\subsection{Modelos existentes de interfaces para la recuperación de información.}

Desde el punto de vista general, considerando ampliamente las características que debe ofrecer una interfaz, partiremos de los trabajos que citaremos a continuación.

Norlin (1992) estudia los modelos de desarrollo de interfaces: individual, informal desestructurado, estructurado consensuado y estructurado jerárquico. 
Esta clasificación se aplica a la responsabilidad y distribución de funciones durante los procesos de desarrollo del interfaces. Norlin insiste en la necesidad e importancia del prototipado de interfaces, así como las ventajas y desventajas que aportan ciertas técnicas tales como menús desplegables, lenguajes de órdenes y ventanas de información.

Por su parte, Marchinioni (1992) indica algunas líneas de actuación en el desarrollo de interfaces. Fundamentalmente, destaca el hecho de que los interfaces deben ser comunes a cualquier fuente de información. También remarca la necesidad de combinar distintas funciones de búsqueda en una interfaz, así como la importancia de incluir en la misma, características de trabajo en grupo y colaboración entre humanos y máquinas. El autor da por supuesta la transparencia y adaptabilidad de una interfaz y la posibilidad de utilizar nuevas técnicas de entrada y visualización de datos (guantes de datos, seguimiento ocular, reconocimiento de voz, etc.). Finalmente, se refiere al desarrollo de interfaces inteligentes, capaces de tutorizar los pasos que realiza el usuario durante una búsqueda, de forma que sea aconsejado y guiado. En definitiva, Marchioni propone el desarrollo de interfaces adaptables (independientemente del nivel y origen cultural del usuario) y activos.

Hay que tener en cuenta que la interfaz debe poseer una serie de características fundamentales. A este respecto, Tague y Schultz (1989) desarrollan los conceptos de Informatividad (capacidad de que el usuario pueda recibir información útil y no repetitiva de los registros consultados), amigabilidad (facilidad de acceso a las diversas funciones del SRI) y visualización de la recuperación (donde todos los elementos visualizados sean oportunos, adecuados y se presenten de un modo agradable).

García Marco (1995) se centra en la temática de los interfaces amigables, detallando el significado de dicho término. Esencialmente indica que la interfaz amigable debe combinar distintos tipos de códigos comunicativos (visuales, auditivos, textuales), estructurar las funciones en niveles jerárquicos de fácil acceso al usuario, propiciar la inferencia metafórica por parte del usuario, ofreciéndole diversos tipos de ayuda durante la utilización de la interfaz.

El tema del interfaz como metáfora es abordado, además, por otros autores como Marchinioni (1992) que también estudia los sistemas de ayuda en la interfaz de los SRI. Uno de los trabajos más interesantes al respecto es el que ha realizado la compañía Inxight, que tuvo su origen en el laboratorio de investigación de Xerox en Palo Alto. Rao (1996) describe cómo estamos ante un nuevo concepto en la relación hombre máquina, en los procesos de visualización de enormes cantidades de información (de un modo visual e interactivo). Además, para este autor, las interfaces adquieren características (sonidos, animaciones, interac- 
tividad, etc.) que permiten al usuario realizar asociaciones entre lo que está experimentando y la realidad que conoce.

Engel (1997) presenta un modelo de interfaz adaptable a las necesidades del usuario dentro de un entorno de trabajo en red, estudiando los aspectos de acceso, certeza de las respuestas obtenidas, aspecto de la interfaz, ayuda recibida por el usuario, adaptabilidad y relevancia de la información que recupera el usuario. Sin embargo, también hay que centrarse en aspectos más concretos, en funcionalidades específicas que deben ofrecer las interfaces en los SRI.

Davis (1992) nos ofrece un esquema de identificación de funciones básicas en SRI de bases de datos en CD-ROM. Este autor se refiere a tres tipos de funciones (básicas, operacionales y de navegación) que integran las bases de este tipo de interfaces. Las características que describen son muy simples, aunque constituyen la base de las distintas posibilidades de las que dispone el usuario para la interrogación de las Bases de Datos.

En cuanto a los OPACs, disponemos de estudios muy detallados (Cherry, 1994), que resultan válidos (en cuanto al planteamiento general) para otro tipo de sistemas de recuperación de información.

Kehoe (1985) enfatiza el hecho de que en muchas ocasiones el usuario no sabe manejar de forma adecuada las diversas herramientas que ofrecen los interfaces de los SRI. En esos momentos, el interfaz debería recurrir a sistemas expertos, que tratarían de guiar y aconsejar al usuario en el uso de las funciones de la interfaz. Denning (1994) incide en este aspecto y añade un nuevo elemento, el intermediario inteligente, entre el usuario y las características de recuperación de información de la interfaz. El intermediario inteligente, permitiría prescindir de intermediarios humanos y evitaría que el usuario tuviese que seguir un proceso de formación, ahorrando los consiguientes costes de tiempo y recursos. Estos agentes inteligentes aprovecharían los resultados de búsquedas anteriores o el seguimiento de las consultas anteriormente realizadas por el usuario; y, a continuación, le propondrían nuevas estrategias de búsqueda por medio del uso de un lenguaje de comandos, de un tesauro o de alguna otra herramienta de consulta.

Algunos autores (Hancock-Bealieu, 1995) presentan una interfaz que aprovecha los resultados para la reformulación de búsquedas a partir de aquellos documentos que el usuario juzga relevantes. Este sistema utiliza los términos más relevantes de los documentos que recupera el usuario.

Uno de los trabajos más interesantes es el de Pejtersen (1991), donde nos presenta un modelo de interfaz basado en la asociación de palabras que se coordinan entre si para formar redes semánticas de unitérminos, estableciendo incluso una tipología de relaciones entre unitérminos, para crear descriptores y tesauros (Pejtersen, 1991, p.13-69). Pejtersen aporta una interfaz de búsqueda que 
utiliza las redes semánticas, en vez del clásico lenguaje de interrogación, para la elaboración de las consultas.

\section{Resultados}

De todo lo anterior podemos deducir que las interfaces en los SRI ofrecen una serie de características que nos ayudan a modelizar diversos aspectos funcionales. Proponemos, pues, las siguientes etapas para el estudio y delimitación de las características de la Interfaz en este tipo de aplicaciones.

\subsection{Arquitectura de acceso}

En primer lugar, hay que tener en cuenta la manera en que se desarrolla la interacción entre el usuario y el SRI, desde el punto de vista de localización de recursos. Será en esta etapa cuando se analizará si el sistema tiene que funcionar en un entorno local o utilizando cualquier tipo de conexión a redes. Además, en esta etapa se atiende también a la identificación de usuarios y control de acceso al sistema.

Es necesario que el SRI realice un control de usuarios, de modo que permita la personalización de la interfaz. Como resultado de esta personalización, en cuanto el usuario se identifique, se recuperarán de manera automática la configuración de la interfaz y las opciones personalizadas.

La interfaz debe permitir la consulta de Bases de datos en modo local (CDROM o Disco Duro) o por medio de una conexión en red (Red de Trabajo en Grupo, TCP/IP, Novel Netware, PC/NFS, etc.) indistintamente. Así pues, la localización de los recursos de información y bases de datos debe resultar transparente al usuario.

\subsection{Interfaz general}

Este aspecto está muy relacionado con la Interfaz Gráfica de Usuario (IGU). Profundizando en su estudio, podremos delimitar las características de generalización. Coincidimos con García Marco (1995) en cuanto a las características que debe reunir la Interfaz general en un SRI, a saber:

- Tipos de códigos comunicativos diferentes (visuales, icónicos, auditivos, textuales), que permitan al usuario identificar y clasificar los elementos de interacción e información entre usuario y sistema.

- Estructuración de las funciones en niveles jerárquicos, fundamentalmente mediante la organización de las distintas opciones y procesos en menús jerárquicos y desplegables. 
- Presencia de elementos metafóricos que permitan al usuario identificar los elementos de la interfaz con objetos presentes en la realidad cotidiana, tales como el escritorio, carpetas, documentos, marcadores, archivadores, etc.

- Existencia de un sistema de ayuda que guíe al usuario en la utilización del sistema.

Actualmente, una interfaz basada en modo texto y que no aproveche las características de los entornos de tipo Windows (menú-ventana-icono-ratón) no tiene sentido, ya que rompe con la necesidad de integración entre el SRI y el resto de aplicaciones del entorno de trabajo del usuario.

\subsection{Adaptación al usuario}

Es necesario estudiar en qué medida la interfaz se adapta a las necesidades, preferencias y gustos de cada persona, detallando aspectos de identificación individualizada de usuarios, posibilidad de guardar perfiles de usuario, personalización de la interfaz general del sistema y de los formatos de salida, e incluso si el sistema nos permite definir procesos de difusión selectiva de información.

El sistema debe ofrecer al usuario la personalización de las opciones disponibles en los menús desplegables, así como la definición de barras de herramientas que permitan el acceso rápido a determinados procesos. También es posible que el usuario quiera personalizar colores, tipos de letra, gráficos de fondo, iconos y la posición y tamaño de las distintas ventanas de la interfaz del SRI.

Además de las funciones de búsqueda de información, la interfaz del SRI debe permitir la definición de estrategias de búsqueda, utilizando diversas herramientas. Estas estrategias serían realmente perfiles de necesidades de información. Tras la identificación por parte del usuario, el SRI actualizaría los resultados de las estrategias de búsqueda e informaría al usuario de los cambios producidos, ya sea en el mismo momento de actualización de las estrategias o a través de un mensaje de correo electrónico.

Por último, queremos señalar la importancia del hecho de que la interfaz sea capaz de proporcionar la adaptación de las funciones de recuperación y consulta de la información. El usuario podrá especificar el formato de visualización de los elementos recuperados, formato de impresión en papel, conservación de los elementos recuperados en un fichero de disco, envío de resultados a direcciones de correo electrónico, etc.

\subsection{Gestión de contenidos}

La pretensión de intentar homogeneizar distintas fuentes de información, para utilizar una misma interfaz de recuperación de información, es impracticable, debido a la naturaleza diversa de contenidos, productores de información o medios de difusión. Así pues, es preferible que el sistema pueda gestionar dife- 
rentes tipos de elementos informativos o bases de datos de forma conjunta o individual. Parece lógico que la interfaz debe estar preparada para ello y nos debe permitir la interacción entre distintas colecciones de información. La interfaz del SRI debe permitir la selección individualizada de colecciones o bases de datos para la realización de procesos de búsqueda. La lista de bases de datos disponibles debe estar fácilmente accesible en todo momento, dentro de un menú desplegable o en una ventana.

El SRI no es únicamente una herramienta de recuperación, sino que estas mismas estructuras sirven para generar las propias bases de datos documentales, adaptadas a los recursos de información gestionados de manera personal por el usuario, y que están localizados en su sistema de almacenamiento local. De este modo la interfaz del SRI, debe considerar aquellos documentos existentes en el disco duro del usuario, CD-ROMs, etc., como elementos de una colección de información documental organizada.

\subsection{Esquemas de búsqueda}

Es indiscutible que el modelo de búsqueda con mayor difusión, es el basado en el uso de un lenguaje de órdenes para la formulación de consultas. Generalmente se utilizan lenguajes que permiten al usuario la introducción de términos, así como combinarlos con operadores lógicos, operadores de adyacencia, e incluso delimitar las búsquedas según el contenido de los campos o atributos de los elementos informativos a recuperar.

También pueden utilizarse redes semánticas o tesauros, para la formulación de consultas a través de la identificación de conceptos, que constituyen auténticos mapas de la información contenida en las bases de datos. El usuario realizaría una selección de términos o descriptores durante el proceso de consulta/navegación del tesauro/red semántica. Dicho proceso se llevaría a cabo sobre una presentación alfabética, basada en grafos o sistemática. Posteriormente, los descriptores seleccionados podrían combinarse entre sí por medio de operadores booleanos.

Otro esquema de búsqueda sería el "browsing" o examen de los glosarios y contenidos de los campos de las distintas bases de datos. El usuario visualizaría una serie de listas o tablas donde aparecen el contenidos de campos concretos. Por ejemplo; realizando un examen sobre el campo "Autor" se obtendría una lista de todos los autores de los elementos informativos de las bases de datos, junto con su frecuencia de aparición. Evidentemente el usuario podría combinar el examen de varios campos para la construcción de las consultas.

La interfaz del SRI podría incluir la selección de términos de búsqueda mediante la consulta de índices permutados KWIC. La elaboración de estos índi- 
ces sería automática y según el contenido de determinados campos seleccionados por el usuario.

Sin embargo existen una serie de problemas, relacionados fundamentalmente con el uso de bases de datos o colecciones de información heterogéneas, y con su integración en un único modelo de búsqueda:

1. La elaboración de consultas donde se utiliza la delimitación de términos según el contenido de ciertos campos.

2. La unificación de tesauros/redes semánticas de diferentes bases de datos y su integración en una única herramienta de consulta.

En el primer caso, la interfaz del SRI debe ofrecer al usuario una gran base de datos virtual, que combine aquellas que ha seleccionado en la gestión de contenido, identificando campos comunes y específicos, de las bases de datos utilizadas. En el segundo caso, habría que reunir los distintos tesauros o redes semánticas individuales de cada base de datos y unificar los diferentes campos semánticos en un único "macrotesauro" o "macrored".

Otra herramienta interesante en la búsqueda de información es la expansión de consultas utilizando los resultados de búsquedas anteriores (HandcockBeaulieu, 1995). Para que el SRI pueda llevar a cabo esta función, la interfaz debe estar preparada para ello. El usuario puede seleccionar elementos individuales de los conjuntos recuperados, y lanzar una nueva búsqueda de ítems informativos relacionados con aquellos que han sido seleccionados.

Una posibilidad de reciente desarrollo sería la creación de agentes de búsqueda. Tras la especificación de una serie de necesidades de información, el agente de búsqueda comenzaría a realizar labores de consulta a las distintas fuentes de información de forma autónoma. Estos procesos se realizarían en segundo plano, sin que interferir en las distintas operaciones que realice el usuario. Las estrategias de búsqueda se elaborarían a partir de la formulación de consultas, independientemente de las herramientas utilizadas. Estas estrategias, tal y como se indica en el punto 3.3., se actualizarían cada vez que el usuario accediese al sistema.

\subsection{Recuperación y consulta}

El proceso que sigue a la búsqueda es la consulta de los elementos recuperados. Existen múltiples modos de visualización de los conjuntos recuperados en general y de cada elemento en particular. Quizá sea éste el momento más crítico durante el uso de los SRI.

Cada conjunto de elementos recuperados debe visualizarse en una ventana con el contenido de algunos campos esenciales (título, año, autor, fuente, etc.). Para evitar la confusión del usuario, debido a la proliferación de ventanas, la 
interfaz debe presentar una ventana con un histórico de las consultas realizadas y el número de elementos recuperados; las distintas consultas deberán llevar algún tipo de marca o icono identificativo, que informe al usuario sobre si una determinada consulta se trata de un agente en progreso y si se ha guardado como estrategia de búsqueda. Una tercera ventana mostrará el contenido del elemento seleccionado en la ventana de elementos recuperados.

Por otra parte, es necesario que el SRI consiga una interacción total entre los procesos de búsqueda y consulta. Para ello la interfaz ha de permitir la expansión de consultas desde las ventanas de resultados y visualización. El aspecto general de la interfaz podría ser el expuesto en el siguiente gráfico (Fig. 3).

La ventana de resultados debe exponer de forma clara y estructurada los elementos recuperados. De este modo se evita que el usuario pueda sufrir una desorientación o un desbordamiento cognoscitivo, debidos fundamentalmente a la gran cantidad de ítems que pueden recuperarse durante el proceso de búsqueda. El método que se sigue comúnmente es la utilización de listas, donde los elementos se ordenan según un determinado criterio (ponderación de los términos de consulta, título, autor, año, etc.).

Ventana de aplicación

\begin{tabular}{|c|c|c|}
\hline \multicolumn{3}{|c|}{ Menús desplegables } \\
\hline $\begin{array}{l}\text { Ventana de } \\
\text { Histórico de } \\
\text { consultas }\end{array}$ & $\begin{array}{l}\text { Ventana de } \\
\text { Resultados } \\
\text { de la consulta } \\
\text { seleccionada en el } \\
\text { Histórico de } \\
\text { consultas }\end{array}$ & $\begin{array}{l}\text { Ventana o } \\
\text { barra de } \\
\text { Herramientas }\end{array}$ \\
\hline \multicolumn{2}{|c|}{$\begin{array}{l}\text { Ventana de visualización del elemento } \\
\text { seleccionado en la Ventana de Resultados }\end{array}$} & \\
\hline \multicolumn{3}{|c|}{ Barra de Estado } \\
\hline
\end{tabular}

Fig. 3. Aspecto general de la Interfaz en un SRI 
Sin embargo, podrían utilizarse presentaciones que ayuden a una mejor comprensión de los resultados por parte del usuario. La utilización de grafos para representar el nivel de generalidad o especialización de los elementos recuperados puede ayudar en la obtención de una visión global de las relaciones de contenido. La interfaz puede mostrar los ítems informativos en forma de estructuras jerárquicas, mediante técnicas de agrupación.

También puede utilizarse el tesauro o red semántica para organizar la presentación de resultados. En este tipo de visualización, los elementos recuperados aparecen ligados a los términos del tesauro, que a su vez están relacionados entre sí, mediante las relaciones de jerarquía y asociación.

\subsection{Sistemas de ayuda al usuario}

Hay que tener en cuenta que la interfaz constituye un elemento activo, que debe reaccionar a las acciones que lleva a cabo el usuario. En el caso de los SRI, debe hablarse de sistemas de ayuda, que deben actuar como "agentes" que ofrecen información referencial sobre el uso de la aplicación. Pero hay que ir más allá, estos agentes deben guiar al usuario en la elaboración de estrategias de búsqueda, mediante la recomendación del uso de esquemas de búsqueda y la reutilización de los resultados para la formulación de nuevas consultas.

Además de una introducción al uso del SRI y de la existencia de un tutorial on-line, la interfaz debe ofrecer una serie de mensajes contextuales de ayuda, que proporcionen información acerca de los aspectos de las estrategias de búsqueda que pueden utilizarse en cualquier momento del uso del sistema.

Por ello, si consideramos el sistema de ayuda del SRI como un elemento activo, ofreceremos al usuario, de forma rutinaria, procedimientos o mensajes que indiquen los posibles pasos a seguir.

Es imprescindible que se proporcione información acerca de la actividad del sistema, cuando un proceso conlleva un tiempo considerable, así como la presentación de mensajes de error cuando el usuario intente realizar algún tipo de operación no permitida, o si el sistema encuentra algún problema para realizar las operaciones solicitadas.

El usuario agradecerá que el SRI ofrezca una utilidad o asistente, cuya finalidad sea la de indicar cómo se desarrollan o realizan determinadas operaciones.

\subsection{Integración con el entorno del usuario}

La integración entre aplicaciones es actualmente un requisito imprescindible para evaluar su calidad, incluyendo a los SRI. El entorno de trabajo, se puede considerar como una única aplicación con diferentes facetas: Procesador de textos, hojas de cálculos, correo electrónico, agenda, bases de datos, navegadores Web, etc. 
De hecho, el éxito imparable del las interfaces de los clientes Web ha sido su capacidad de asumir los diferentes protocolos de Internet, y el acercamiento de las tecnologías de comunicación en red al entorno ofimático.

La obtención de resultados en un SRI no es el final del proceso de búsqueda y recuperación de información, sino que aporta al usuario la información que necesita para que con posterioridad la pueda integrar en sus entorno de trabajo individual, generando documentos, comunicando los resultados y accediendo a los documentos finales.

Cualquier tipo de información obtenida en un SRI debería poderse almacenar en un formato electrónico, adaptable a las herramientas manejadas por el usuario: ficheros de texto, hojas de cálculo, ficheros gráficos, bases de datos, documentos HTML, mensajes de correo electrónico. Es fundamental la existencia de opciones para exportar datos, el uso de una utilidad de portapapeles compartido por todas las aplicaciones del entorno de trabajo y la posibilidad de la manipulación de objetos entre aplicaciones. Por ejemplo, si un usuario, arrastra un conjunto de elementos recuperados a una dirección de correo electrónico de nuestra agenda personal, se enviarán dichos resultados automáticamente.

No olvidemos que es prioritario, y a veces olvidado, el poder imprimir resultados con calidad, orden y flexibilidad. La salida impresa es uno de los factores que los usuarios más valoran para uso personal y comunicación.

Toda búsqueda de información tiene como objetivo el acceso final a los documentos. Cada vez más los SRI, además de constituirse en una herramienta referencial, deben integrar en su interfaz el acceso al texto completo de los documentos. Internet es un medio en el que se mezcla lo referencial con lo textual de manera desordenada, pero integrados en un mismo esquema de navegación. Un SRI debe contemplar la navegación/consulta de los documentos a los que hace referencia, en un formato integrado con el del resto de aplicaciones del usuario (PDF, MS-Word, Documentos CAD, HTML, etc.). Aunque a veces se consideren como etapas separadas el acceso a las funciones del SRI han de permanecer visibles mientras se consultan los documentos.

\subsection{Estructuración de los aspectos del modelo de interfaz}

Los aspectos de la Interfaz desarrollados anteriormente deben estructurarse para su comprensión dentro de los procesos de recuperación de información. Es evidente que, al proponer un modelo horizontal (donde se adopta una arquitectura basada en facetas y no en niveles), existen múltiples posibilidades de estructuración. Es este trabajo, nos hemos decantado por aquel que está más cercano al usuario, desde el punto de vista de interacción con el SRI, tal y como se muestra en el siguiente gráfico (Fig. 4). 
Cuando un usuario comienza a utilizar el sistema, realiza, en primer lugar, una serie de operaciones que le permiten el acceso a los contenidos. A continuación, se produce una toma de contacto, entre el usuario y el interfaz general, de la que se deduce la integración entre su entorno de trabajo y el SRI. A partir de ese momento, se entra en una dinámica de utilización de las diversas operaciones de búsqueda, gestión de contenidos, utilización de sistemas de ayuda y las posibilidades de adaptación del SRI al usuario, para finalizar con la recuperación y consulta de los elementos de información.

\section{Conclusiones}

- La arquitectura de acceso al SRI debe estar en función de su entorno, local o con posibilidades de conexión a redes.

- El modelo de interfaz debe adaptarse a las necesidades y preferencias de los diferentes tipos de usuarios mediante la estructuración de accesos a procesos, uso de metáforas, etc., que permitirán la recuperación de información de forma personalizada.

- Debe facilitar la interacción entre las diferentes bases de datos a las que permite acceder el sistema, con independencia de la naturaleza, formato y contenido de los elementos informativos.

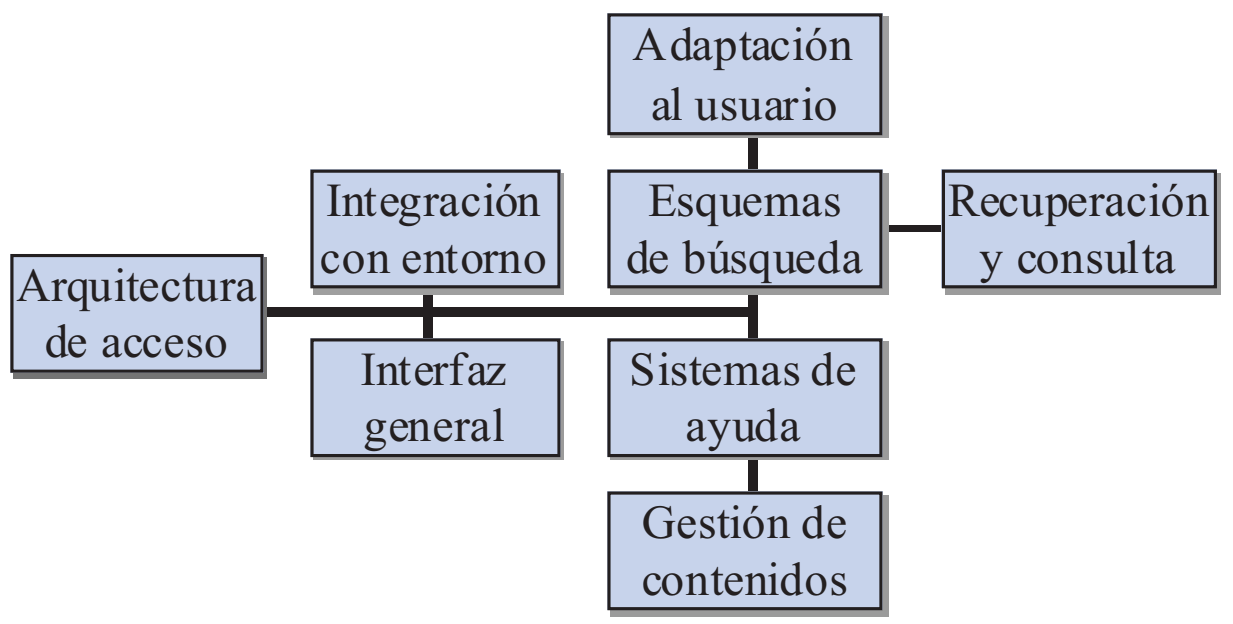

Fig. 4. Estructuración de las facetas del modelo de Interfaz

Scire. $4: 1$ (en.-jun. 1998) 71-88 
- La selección de esquemas de búsqueda y los formatos alternativos para la visualización de los conjuntos recuperados son tan importantes en el diseño de la interfaz como la capacidad de dicho interfaz para comunicar al usuario las posibilidades de las que dispone para integrarlo con otras aplicaciones.

\section{Referencias}

Abad García, M. F. (1997). Investigación evaluativa en documentación : aplicación a la documentación médica. // Valencia : Universidad, 1997.

Blair, D. C. (1990). Language and representation in information retrieval. // Amsterdam : Elsevier Science Publishers, 1990.

Catenazzi, N. ; Sommaruga, L. (1994). Hyper-Book : A formal model for electronic books. // Journal of Documentation 50 :4 (Dec. 1994) 316-332.

Cherry, J. M. ; y otros (1994). OPACs in Twelve Canadian Academic Libraries : An Evaluation of Functional Capabilities and Interface Features. // Information Technology and Libraries. (Sep. 1994) 174-195.

Croft, W. B. ; Turtle, H. (1989) A retrieval model for incorporating hypertext links. // Hypertext ' 89 Proceedings. ACM : New York, 1989. P. 213-224.

Davis, D. (1992). Evaluation of SIGCAT's CD-ROM Consistent Interface Guidelines, and US standards and draft standards affecting distributed computer applications. // Online Information 92. Londres, 1992.

Engel, G. (1997). A Personal Portable Information Interface : A Model for Meeting User Needs in the Networked Information Environment. // Cataloging \& Classification, 24 : 2 (1997) 141-161.

García Marco, F. J. (1995). Interfaces amigables para la recuperación de la información bibliográfica. // SCIRE $1: 1$ (en.-jun, 1995) 127-148.

Handcock-Beauleu, M. ; Fieldhouse, M. ; Do, T. (1995). An Evaluation of Interactive Query Expansion in an Online library catalogue with a graphical user interface. // Journal of Documentation. 51 : 3 (1995) 225-243.

Kerr, S. T. (1987). De la página a la pantalla. // Lambert, S.; Ropiequet, S. (eds.). CD ROM : El nuevo papiro. Madrid : Anaya, 1987. P. 339-366.

Lancaster, F. W. (1978) Precision and recall. // Encyclopedia of Library and Information Science. 1978. Vol. 23, p. 170-180.

Lee, K.; Lee, Y. K.; Berra, P. B. (1997). Management of Multi-structured Hypermedia Documents : A Data Model, Query Language, and Indexing Scheme. // Multimedia Tools and Applications. $4: 2$ (1997) 107-131.

Manglano Bosch, V. ; Handcock-Beauleu, M. (1995). CD-ROM user interface evaluation : approachtiness of IE. // Online \& CD-ROM Review. 19 : 5 (1995) 255-270.

Marchinioni, G. Interfaces for End-User Information Seeking. // Journal of the American Society for Information Science. $43: 2$ (1992) 156-163.

Martínez, J. M.; Hilera, J. R. (1998). Modelado de documentación multimedia e hipermedia. // Cuadernos de Documentación Multimedia. 6 (1998) 211-220.

Scire. 4 : 1 (en.-jun. 1998) 71-88 
Norlin, A. ; y otros (1992). Interface design and Development. // Library Hi Tech. 10 : 3. (1992) 7-24.

Pastor, J. A. ; Saorín, T. (1995.). El hipertexto documental como solución a la crisis conceptual del hipertexto : el reto de los documentos corporativos en redes. // Cuadernos de Documentación Multimedia. 4 (1995) 41-55.

Pejtersen, A. M. (1991). Interfaces Based on Associative Semantics for Browsing in Information Retrieval. // Riso National Laboratory : Roskilde, 1991.

Rada, R. ; y otros (1991). Collaborative hypertext and the MUCH system. // Journal of Information Science. 17 (1991) 191-196.

Tague, J.; Schultz, R. (1989). Evaluation of the user interface in an information retrieval system : A model. // Information Processing \& Management. 25 : 4 (1989) 377-389.

Wright, P. (1989). Interface Alternatives for Hypertext. // Hypermedia. 1 : 2 (1989) 146166. 